\title{
Platelet concentration and its effect on bone formation in calvarial defects: An experimental study in rabbits
}

\author{
Eun-Seok Kim, DDS, MS, ${ }^{a}$ Eun-Jin Park, DDS, MS, ${ }^{b}$ and Pill-Hoon Choung, DDS, MS, PhDc \\ Chungnam National University Hospital, Taejeon, and College of Dentistry, Seoul National University, \\ Seoul, Korea
}

\begin{abstract}
Statement of problem. The use of the platelet concentration technique is widespread in dental implant surgery. However, its effect or mechanism is not clearly understood.

Purpose. This study introduced an animal model for the platelet concentration technique and evaluated its effect on bone formation with natural cancellous bovine bone mineral.

Material and methods. Adult New Zealand white rabbits were used as the animal model. A density gradient medium was used to obtain a constant platelet count for the preparation of platelet concentrates. In the experimental group, natural cancellous bovine bone mineral with added platelet concentrates was grafted onto critically sized bony defects of the rabbit calvarium. Bone formation in the tissue sections was evaluated with soft $\mathrm{x}$-ray imaging and computer tomography.

Results. The average platelet count of the rabbit platelet concentrates was $1487 \times 10^{3} / \mu \mathrm{L}(287 \%$ concentrated). In all the tested parameters, greater bone densities were obtained in grafts that were combined with platelet concentrates.

Conclusion. This study showed that the rabbit is a useful animal model for studying the platelet concentration technique. When combined with grafts of natural cancellous bovine bone mineral, the technique increased bone formation. (J Prosthet Dent 2001;86:428-33.)
\end{abstract}

\section{CLINICAL IMPLICATIONS}

In this experimental rabbit study, platelet concentrates combined with grafts of natural cancellous bovine bone mineral increased bone formation.

n dental implant surgery, the regeneration of highquality bone is very important but difficult to attain. Although many natural or recombinant growth factors are introduced to increase bone formation, their clinical use is limited because of the cost and/or immunologic problems.

In 1994, Tayapongsak et al $^{1}$ reported that autologous fibrin adhesive (AFA) extracted from cryoprecipitation enhanced new bone formation in an autologous cancellous bone graft; this effect was attributed to the fibrin network. Several years later, Marx et $\mathrm{al}^{2}$ reported that platelet-rich plasma (PRP) (or more precisely, platelet

Presented at the May 1999 1st Biennial Congress of the Asian Academy of Prosthodontics in Seoul, Korea; winner of the Hiranuma Award (best oral presentation).

This work was supported by the Korean Science and Engineering Federation through the Intellectual Biointerface Engineering Center at Seoul National University.

aAssistant Professor, Department of Oral and Maxillofacial Surgery, Chungnam National University Hospital.

bProsthodontist, private practice, Taejeon, Korea.

cProfessor, Department of Oral and Maxillofacial Surgery, Craniofacial Tissue Engineering Laboratory of BK 21 Human Life Science and the Intellectual Biointerface Engineering Center, College of Dentistry, Seoul National University. concentrates) obtained by means of a cell separator enhanced new bone density in a mandibular reconstruction when placed with an autologous cancellous bone graft. It was reported that PRP had a radiographic maturation rate 1.62 to 2.16 times that of bone grafts without it; a greater bone density also was observed over the same period. The beneficial effect of the platelet concentrates was attributed to local growth factors such as the platelet-derived growth factor (PDGF) and transforming growth factor- $\beta$ (TGF- $\beta$ ) contained in the platelet. Additional advantages of the platelet concentrates include their adhesive nature (stabilization of the graft material), hemostasis, and lack of an immune reaction.

In addition to PDGF and TGF- $\beta$, osteonectin (a major protein of mineralized bone) is an alpha-granule component. ${ }^{3}$ Moreover, platelets contribute to the circulating level of bone sialoprotein, which is a major bone-related protein. ${ }^{4}$ This implies that platelet concentrates can influence bone formation through a variety of pathways.

Since Marx's report, the platelet concentration technique has enjoyed widespread use in implant dentistry. However, its precise effect or mechanism of action is unknown. The development of an animal 


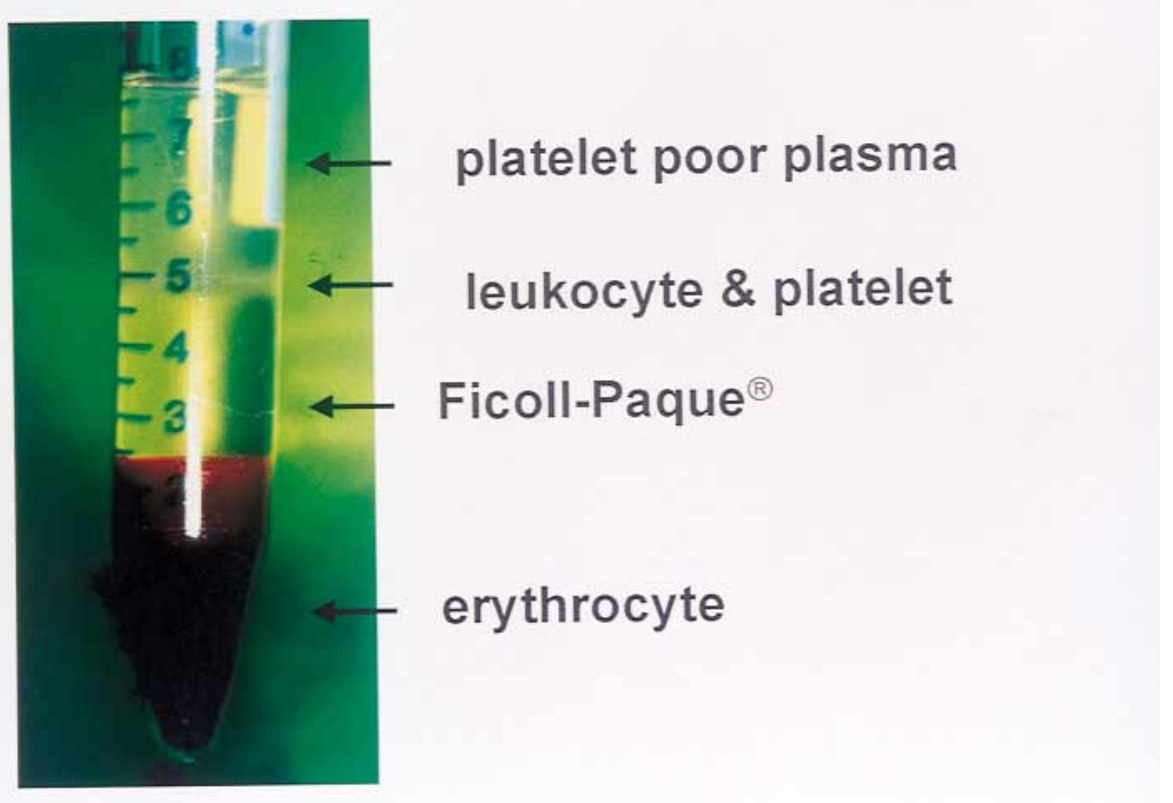

Fig. 1. Four layers after first centrifuge ( $400 \mathrm{~g}$ for 40 minutes). Leukocyte and platelet layers were more turbid than Ficoll-Paque or platelet-poor plasma.

model would be of great assistance in understanding the role of the growth factors in platelet concentrates and their effect on bone formation. The purposes of this study were as follows: (1) to introduce an animal model for evaluating the platelet concentration technique, and (2) to determine the osteoinductive or osteoconductive potential of a mixture of platelet concentrates and natural cancellous bovine bone mineral in calvarial bony defects.

\section{MATERIAL AND METHODS}

Twenty New Zealand white rabbits that were fed on a standard rabbit diet and weighed, on average, $3.0 \pm 0.4 \mathrm{~kg}($ mean $\pm \mathrm{SD})$ were selected as the animal model. Fresh blood treated with $8 \mathrm{~mL}$ acid citrate dextrose anticoagulant solution $(\mathrm{ACD}, 0.1 \mathrm{~mL} / \mathrm{mL}$ ) was obtained from the intermedial branch of the caudal auricular vein of the rabbits.

A constant platelet count was obtained. The degree of selective purification of cell populations from the blood affects the validity of subsequent experimental data. Techniques for the selective purification of cell populations have included gradient centrifugation, electrophoresis (adhered to various charge surfaces), in vivo irritant-induced cell concentration, and antibody lysis of unwanted populations. ${ }^{5,6}$ Among these, isolation with a gradient material (Ficoll-Paque; Amersham-Pharmacia Biotech Co, Piscataway, N.J.) was chosen for this study. Ficoll-Paque is an aqueous solution consisting of $5.7 \mathrm{~g}$ Ficoll 400 and $9 \mathrm{~g}$ sodium diatrizoate with calcium EDTA per $100 \mathrm{~mL}$ and a density of $1.077 \pm 0.001$ $\mathrm{g} / \mathrm{mL}$. Both the density and osmolarity of FicollPaque have been optimized so that lymphocytes from whole blood can be isolated. Ficoll-Paque is sterile and nontoxic, so it was appropriate for this in vivo study of animals.

Six milliliters of the medium was added to a $15-\mathrm{mL}$ centrifuge tube (Amersham-Pharmacia Biotech Co), and the blood sample was layered on the medium. The tube was centrifuged at $400 \mathrm{~g}$ and $18^{\circ} \mathrm{C}$ to $20^{\circ} \mathrm{C}$ for 40 minutes (Fig. 1). The upper layer was transferred with a clean pipette to a $2-\mathrm{mL}$ centrifuge tube and centrifuged at $5000 \mathrm{~g}$ at $20^{\circ} \mathrm{C}$ for 15 minutes. After removal of the supernatant, $\mathrm{l}$ to $2 \mathrm{~mL}$ PRP was obtained. Both the prepared platelet concentrates and the whole blood of the rabbits underwent platelet number counting by automated hematology (Dilutor STKS-2A; Coulter Corp, Miami, Fla.).

\section{Graft procedure}

After the platelet concentration was prepared, the rabbits were divided randomly into 2 groups. Housing and handling of the animals were in compliance with the Guide for the Care and Use of Laboratory Animals published by the Korea Food and Drug Administration (KFDA Publication, revised Feb. 2000). Each rabbit was injected with $4 \mathrm{mg} / \mathrm{kg}$ gentamycin (Choongwae Pharm Corp, Seoul, Korea) before surgery and for 3 days after surgery. A combination of ketamine hydrochloride $(10 \mathrm{mg} / \mathrm{kg}$, Ketalar, Yuhan, Korea) and $2 \%$ xylazine hydrochloride $(0.15 \mathrm{~mL} / \mathrm{kg}$, Rumpun, Byel, Korea) was given intramuscularly 30 minutes before surgery. 

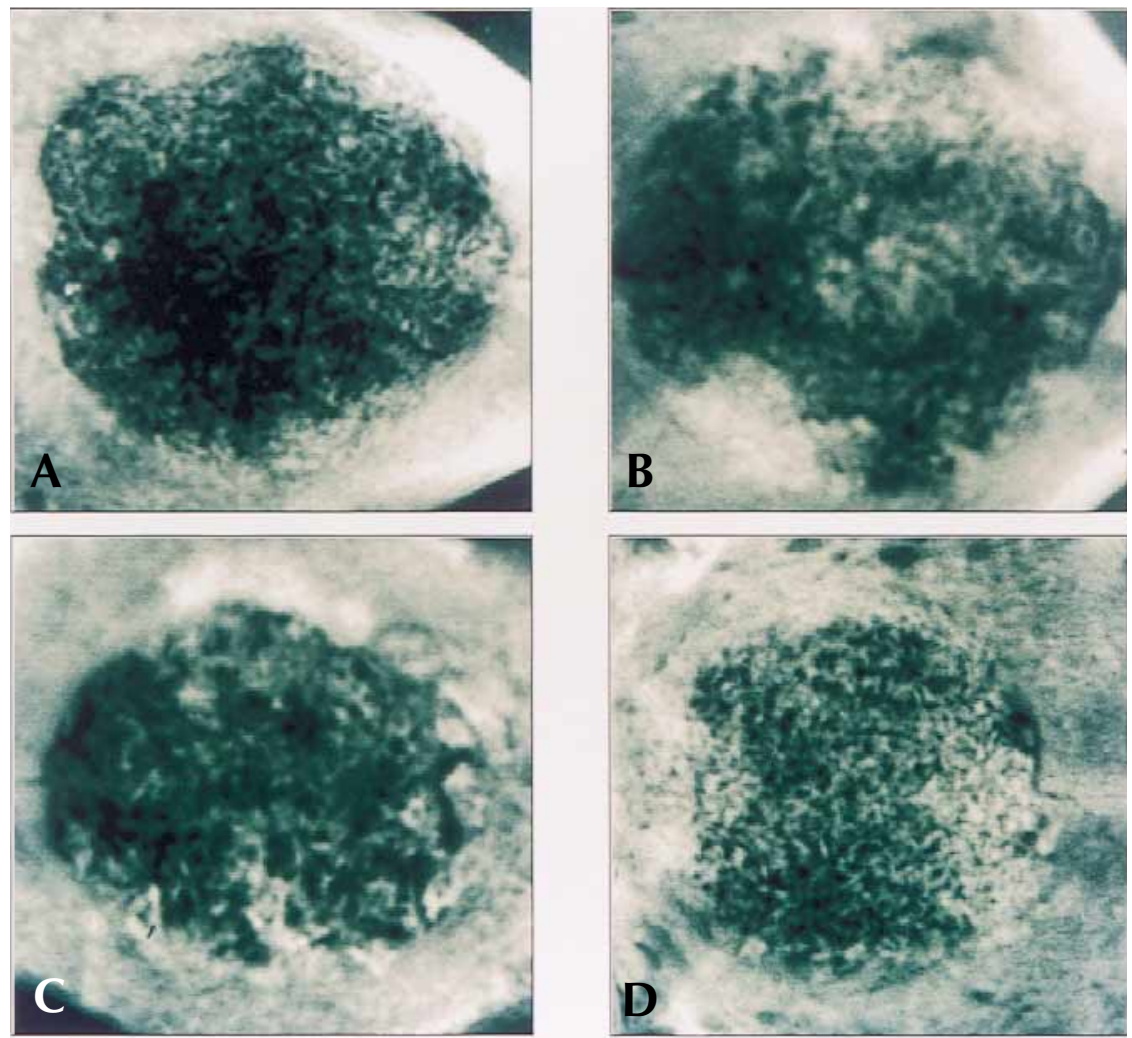

Fig. 2. Soft x-ray images of tissue section. A, Four weeks without platelet concentration. B, Four weeks with platelet concentration. C, Eight weeks without platelet concentration. D, Eight weeks with platelet concentration. More condensed bone formation was observed at periphery of defect in group treated with platelet concentration.

The surgical site was shaved, disinfected with $0.5 \%$ aqueous chlorhexidine di-gluconate (Daewoong Pharm Corp, Seoul, Korea), and then infiltrated with $2 \%$ lidocaine ( $1: 80,000$ epinephrine). After reflection of a subperiosteal flap, a 15 -mm bicortical circular defect was prepared in the calvaria of the rabbits. In the experimental group (group I), the defect was filled with a mixture of platelet concentrates and natural cancellous bovine bone mineral (Bio-Oss; Osteohealth, Shirley, N.Y.) that coagulated with $10 \%$ calcium chloride and human plasma thrombin (Greenplast, Green Cross, Seoul, Korea). In the control group (group II), only natural cancellous bovine bone mineral was used. Double layer sutures were performed with catgut and 4-0 black silk, which were removed 1 week after the surgical intervention.

\section{Evaluation}

According to the established animal care protocol published by the US National Institute of Health, the animals were killed with an overdose of pentobarbital (Choongwae Pharm Corp) at 4 and 8 weeks. After the animals' death, bicortical rectangular bony blocks $(20 \times 20 \mathrm{~mm})$, including the original surgical defect, were prepared.

Contact radiographs of the blocks were produced with a soft $\mathrm{x}$-ray equipment (Cabinet X-ray, Hewlett Packard, Palo Alto, Calif.) at 35 kilovolt (peak) $(\mathrm{kV}[\mathrm{p}])$ for 12 seconds. The $\mathrm{x}$-ray images were recorded on x-ray film (Scopix, Kodak, Rochester, N.Y.), and the radiographs were transformed into digitized images with a color scanner and a standard PC computer. There was no attempt to adjust or calibrate the optical density. The image analysis was performed on a Macintosh computer (Apple, Cupertino, Calif.) with an image analyzer system (Leica Q600, Buffalo, N.Y.). The mineralized area of the defects in the digitized $\mathrm{x}$ rays was identified by the value of the pixel in the images and marked as a region of interest (ROI) around which a $15-\mathrm{mm}$ circle was drawn. The distinction between mineralized and nonmineralized tissue was made by visual evaluation of the gray scale in each image. In addition, the percentage of areas of the mineralized tissue to the ROI were measured.

To compare the integrity and maturation of the graft between the 2 groups, cross-sectional computed tomographic (CT) images of specimens in the center 
Table I. Platelet count: $287 \%$ increase $\left(\times 10^{3} / \mu \mathrm{L}\right)$

\begin{tabular}{lc}
\hline Baseline & Platelet concentrates \\
\hline 518 & 1487 \\
$(502-534)$ & $(1141-1632)$ \\
\hline
\end{tabular}

Table II. Radiopaque area of the soft $x$-ray image analysis $(P<.05)$

\begin{tabular}{lccc}
\hline & & Group I & Group II \\
\hline Soft x-ray & $4 \mathrm{wk}$ & $54.7 \% \pm 5.9 \%$ & $38.3 \% \pm 6.5 \%$ \\
Image analysis & $8 \mathrm{wk}$ & $77.4 \% \pm 4.9 \%$ & $51.0 \% \pm 4.0 \%$ \\
\hline
\end{tabular}

Group I = Natural cancellous bovine bone mineral with platelet concentrates. Group $I I=$ Natural cancellous bovine bone mineral only.

of the calvaria were obtained. In addition, the Hounsfield unit (also called an attenuation value or CT number) was measured at 5 randomly chosen points both in the original defects (graft site) and outside the defects (normal host bone). The CT number of a given material is defined as the relative coefficient of that material resulting from the effective linear attenuation coefficient of water, multiplied by a factor of 1000. The CT numbers for air, water, and dense bone are $-1024,0$, and 1000 , respectively. 8 The maturation or integrity score of the grafts was calculated as a percentage of the normal calvarial bone. Data from image analyses and the CT number were expressed as mean \pm SD for each group. The null hypothesis test was applied at a 5\% significance level with the Wilcoxon rank sum test assuming unpaired samples.

For fluorescent bone labeling, $90 \mathrm{mg} / \mathrm{kg}$ xylenol orange (Sigma, St Louis, Mo.) and $30 \mathrm{mg} / \mathrm{kg}$ calcein blue (Sigma) were parenterally injected at 3 and 7 weeks after surgery, respectively, through the intermedial branch of the caudal auricular vein of the rabbit after sedation with $2 \%$ xylazine hydrochloride. The harvested sample, fixed in $10 \%$ buffered formalin, then was transferred to $70 \% \mathrm{EtOH}$. Thirty- $\mu \mathrm{m}$-thick undemineralized tissue sections were cut with a diamond microtome (RM2155; Leica Instruments Gmbh, Nussloch, Germany).

The sections were viewed at $\times 200$ magnification with fluorescence-labeled microscopy (Olympus BX50F3, Olympus Optical, Tokyo, Japan) with a narrow-band, blue-light filter block (450- to 490-nm barrier, $510 \mathrm{~nm}$ excitation filter).

\section{RESULTS}

The platelet counts of each rabbit yielded a mean value of $5.18 \pm 0.16 \times 10^{5} / \mu \mathrm{L}(502,000 / \mu \mathrm{L}$ to $534,000 / \mu \mathrm{L})$. The mean platelet count of the platelet concentrates was $1.49 \pm 0.35 \times 10^{6}$
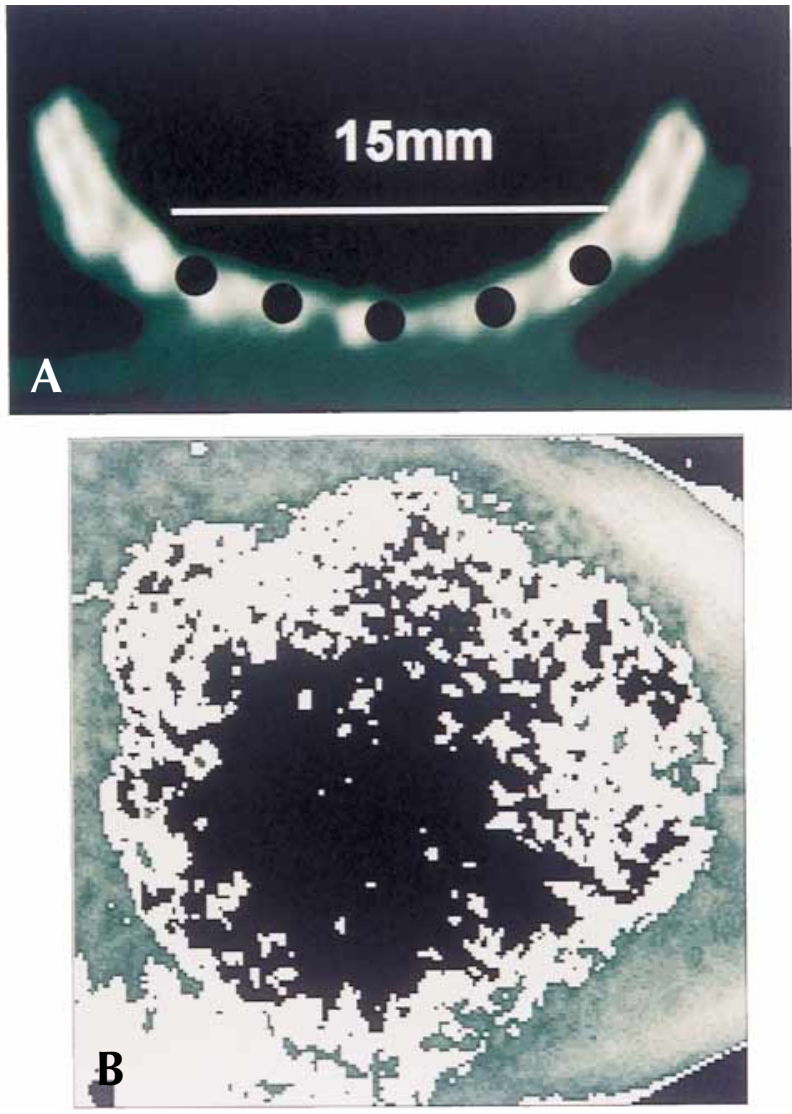

Fig. 3. A, Location of 5 cursors for calculating Hounsfield unit from CT image. B, Digitized format of soft x-ray image of defect for analysis. Radiopaque area of circular defect was calculated automatically.

$(1,141,000 / \mu \mathrm{L}$ to $1,632,000 / \mu \mathrm{L})$. Thus, a $287 \%$ increase in platelet count was observed (Table I). The soft $\mathrm{x}$-ray images showed greater radiopaque and mature bone formation in the experimental group (treated with platelet concentrates) than in the control group (Fig. 2).

In defects of the experimental group, the percentage mineralized area was $54.7 \% \pm 5.9 \%$ at 4 weeks and $77.4 \% \pm 4.9 \%$ after 8 weeks; in the control defects, the area was only $38.3 \% \pm 6.5 \%$ at 4 weeks and $51.0 \% \pm 4.0 \%$ after 8 weeks $(P<.05)$. Thus, the mineralized areas were $16.4 \%$ and $26.4 \%$ larger in the test group than in the control group after 4 and 8 weeks, respectively (Table II, Fig. 3, B). In Figure 3, $B$, white represents the mineralized area and black represents the fibrous connective tissue area. The percentage Hounsfield units of the cross-sectional images showed a relative maturation degree compared with the adjacent normal bone (Fig. 3, $A$ ). In the experimental group, the mean percentage Hounsfield units were $85.8 \% \pm 5.9 \%$ at 4 weeks and $94.7 \% \pm 6.5 \%$ after 8 weeks; in the control group, 

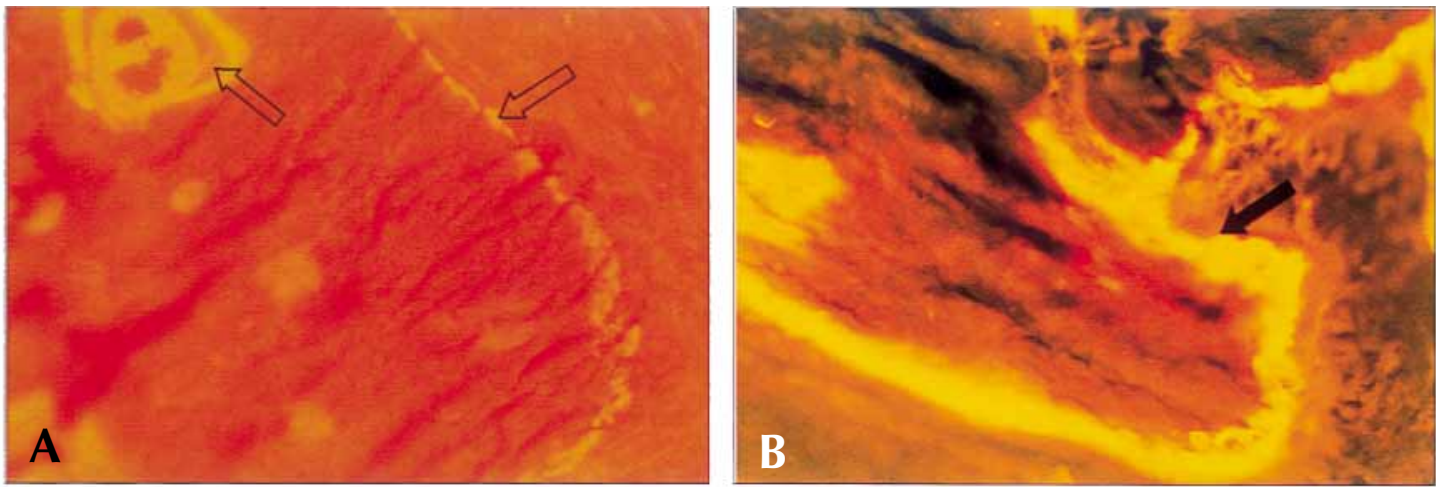

Fig. 4. Fluorescent labeling of xylenol orange at 4 weeks (original magnification, $\times 400$ ). A, Control group treated without platelet concentration. B, Experimental group treated with platelet concentration. More active mineralization was observed in experimental group.

Table III. Percentage Hounsfield units of the cross-sectional images $(P<.05)$

\begin{tabular}{lcc}
\hline & Group I & Group II \\
\hline Computed tomography & (compared with normal bone) \\
$4 \mathrm{wk}$ & $85.8 \% \pm 5.9 \%$ & $57.1 \% \pm 4.0 \%$ \\
$8 \mathrm{wk}$ & $94.7 \% \pm 6.5 \%$ & $76.7 \% \pm 5.6 \%$ \\
\hline
\end{tabular}

Group $I$ = Natural cancellous bovine bone mineral with platelet concentrates. Group $I I=$ Natural cancellous bovine bone mineral only.

they were $57.1 \% \pm 4.0 \%$ at 4 weeks and $76.7 \% \pm 5.6 \%$ after 8 weeks $(P<.05$, Table III).

In the undermineralized tissue sections, the orange and blue fluorescence-labeled surface of the experimental group was thicker than that of the control group (Fig. 4). However, the mineralizing surface of the calcein blue was thinner than the xylenol orange. This may be due to the reduced level of new bone formation or remodeling.

\section{DISCUSSION}

Rabbits are useful animal models for the preparation of platelet concentrates. Generally, the platelets of human and various mammalians (including rabbits) have a similar ultrastructure and constituents. ${ }^{9,10}$ Advantages of using a rabbit as an animal model include the following: (1) easy manipulation, (2) sufficient volume of blood for the preparation of platelet concentrates, (3) survival of platelets similar to the metabolic activity of bone (one third of human), and (4) a high cross-reactivity of antihuman antibodies for immunohistochemical studies.

A $287 \%$ increase in platelet concentrates was obtained from rabbits reproducibly through the use of Ficoll-Paque. This increase was obtained at centrifugal speeds lower than those reported by Marx. ${ }^{2}$ The centrifugal speed can influence platelet activation and trigger platelet secretory processes to the plasma during the preparation of platelet concentrates. A lower centrifugal speed reduces the chance of this occurring. ${ }^{11}$

Several methods exist for assessing new bone formation; these include histomorphometry from image analysis, an assay of the alkaline phosphatase activity, direct photon absorptiometry, and dual x-ray absorptiometry (DEXA). In this study, evaluating the stability of the graft (glue effect) as well as its maturation was important; thus, radiographs of same gray scale were analyzed and CT numbers of the specimens were obtained. These 2 methods allowed an increment in the mineralized area or a decrement in the fibrous connective tissue area to be detected. They did not, however, provide any information about the amount of new bone formed or osteogenesis activity.

The platelet concentrates used in this study provided evidence of enhanced bone formation in natural cancellous bovine bone mineral grafts as well as in autogenous bone grafts. After 8 weeks, the experimental group showed the same mineral density to host cranial bone $(94.7 \%)$. Furthermore, fluorochrome labeling showed the more active bone formation in the experimental group at 4 and 8 weeks. These results are similar to those reported by Marx. ${ }^{2}$

\section{CONCLUSIONS}

This study demonstrated that the rabbit is a useful animal model for evaluating the effect of platelet concentrates. The gradient medium allowed (1) a large number of functionally viable platelets to be purified reproducibly in a relatively short time, and (2) the use of a low centrifugal speed. Further investigation into the effect of platelet concentrates should be undertaken. 


\section{REFERENCES}

1. Tayapongsak P, O'Brien DA, Monteiro CB, Arceo-Diaz LY. Autologous fibrin adhesive in mandibular reconstruction with particulate cancellous bone and marrow. J Oral Maxillofac Surg 1994;52:161-5; discussion 166.

2. Marx RE, Carlson ER, Eichstaedt RM, Schimmele SR, Strauss JE, Georgeff KR. Platelet-rich plasma: growth factor enhancement for bone grafts. Oral Surg Oral Med Oral Pathol Oral Radiol Endod 1998; 85:638-46.

3. Clezardin P, Malaval L, Morel MC, Guichard J, Lecompte T, Trzeciak MC, et al. Osteonectin is an alpha-granule component involved with thrombospondin in platelet aggregation. J Bone Miner Res 1991;6:1059-70.

4. Chenu C, Delmas PD. Platelets contribute to circulating levels of bone sialoprotein in human. J Bone Miner Res 1992;7:47-54.

5. Hoffman M, Monroe DM, Roberts HR. A rapid method to isolate platelets from human blood by density gradient centrifugation. Am J Clin Pathol 1992;98:531-3

6. Perper RJ, Zee TW, Mickelson MM. Purification of lymphocytes and platelets by gradient centrifugation. J Lab Clin Med 1968;72:842-8.

7. Yang KH. Utilization and care of experimental animal. User's guide. 1st ed. Seoul, Korea: National Institute of Toxicology Research in KFDA; 2000. p. 81-8.

8. Curry, TS, Dowdey JE, Murry RC. Christensen's physics of diagnostic radiology. 4th ed. Philadephia (PA): Lea \& Febiger; 1990. p. 307-9.
9. Jain NC. Essentials of veterinary hematology. 1st ed. Philadelphia (PA): Lea \& Febiger; 1993. p. 105-32.

10. Clemmons RM, Bliss EL, Dorsey-Lee MR, Seachord CL, Meyers KM. Platelet function, size and yield in whole blood and in platelet-rich plasma prepared using differing centrifugation force and time in domestic and food-producing animals. Thromb Haemost 1983;50:838-43.

11. Fijnheer R, Pietersz RN, de Korte D, Gouwerok CW, Dekker WJ, Reesink $\mathrm{HW}$, et al. Platelet activation during preparation of platelet concentrates: a comparison of the platelet-rich plasma and the buffy coat methods. Transfusion 1990;30:634-8.

Reprint requests to:

Dr Pill-HoOn ChOUnG

Department of Oral and Maxillofacial Surgery

College of Dentistry, Seoul National University

28-2 YeONGUN-DONG, CHONGRO-KU

SEOUL, KOREA

FAX: (82)2-745-3477

E-MAIL: choungph@snu.ac.kr

Copyright (C) 2001 by The Editorial Council of The Journal of Prosthetic Dentistry.

0022-3913/2001/\$35.00 + 0. 10/1/115874

doi:10.1067/mpr.2001.115874
Noteworthy Abstracts of the Current Literature
Immediate loading of Brånemark System implants following placement in edentulous patients: A clinical report Horiuchi K, Uchida H, Yamamoto K, Sugimura M. Int J Oral Maxillofac Implants 2000;15:824-30.

Purpose. Osseointegration is a condition of clinical immobility of endosseous implants. To achieve osseointegration, a state of undisturbed healing has been described as essential. Recent reports suggest that implants may be capable of withstanding functional loading immediately after surgical placement. The purpose of this study was to evaluate the ability of Branemark System implants to support screw-retained provisional prostheses immediately after surgical placement.

Material and methods. Fourteen patients were treated with implant placement in the edentulous mandible $(n=12)$ or edentulous maxilla $(n=5)$ during the course of this study. Three of the patients received implants in both jaws. At the time of implant placement, the torque required to fully seat the implants was recorded. All implants with an insertion torque of $40 \mathrm{Ncm}$ or greater were used to support dental prostheses immediately after placement surgery. Implants that failed to achieve $40 \mathrm{Ncm}$ of insertion torque or implants that required bone grafting were not immediately loaded. Prosthesis connection consisted of rigid attachment of the implants to a provisional prosthesis with the use of autopolymerizing acrylic resin. After a healing period of 4 to 6 months, a definitive prosthesis was fabricated. At that time, implant immobility was assessed for the immediately loaded and submerged implants.

Results. A total of 140 implants achieved an insertion torque of $40 \mathrm{Ncm}$ and subsequently were used to support prostheses immediately after implant placement. Of these implants, 136 achieved and maintained osseointegration during the study period of 8 to 24 months. All submerged implants $(\mathrm{n}=17)$ were found to be osseointegrated.

Conclusion. The high rate of osseointegration found in implants that were loaded immediately after surgical placement suggests that this treatment may be a viable option for the management of complete edentulism. High initial insertion torque and rigid implant splinting were described as critical for implant integration. 16 References. -SE Eckert 